\title{
Editorial Summary to selected papers from the 2020 IMEKO TC19 International Workshop on Metrology for the Sea - "Learning to measure sea health parameters"
}

\author{
Silvio Del Pizzo ${ }^{1}$ \\ ${ }^{1}$ Department of Science and Technologies, University od Naples "Parthenope", Centro Direzionale Isola C4, Naples, Italy
}

Section: EDITORIAL

Citation: Silvio Del Pizzo, Editorial Summary to selected papers from the 2020 IMEKO TC19 International Workshop on Metrology for the Sea - "Learning to measure sea health parameters", Acta IMEKO, vol. 10, no. 4, article 2, December 2021, identifier: IMEKO-ACTA-10 (2021)-04-02

Received December 14, 2021; In final form December 14, 2021; Published December 2021

Copyright: This is an open-access article distributed under the terms of the Creative Commons Attribution 3.0 License, which permits unrestricted use, distribution, and reproduction in any medium, provided the original author and source are credited.

Corresponding author: Silvio Del Pizzo, e-mail: silvio.delpizzo@uniparthenope.it

\section{Dear Readers,}

This issue of Acta IMEKO is dedicated to the works that have been selected from those presented at "2020 IMEKO TC19 International Workshop on Metrology for the Sea", shortly named MetroSea. The edition 2020 was hosted by the University of Naples "Parthenope", but unfortunately all sessions, discussions and other activities were carried out online due to the pandemic situation. The virtual workshop was organized to broadcast live all sessions according to the conference program, and the attendees were able to participate to all proposed activities by entering in virtual rooms. Therefore, the online conference was not so different from a live event, with the great advantage that every session was recorded and shared with the participants.

The sea is the medium that allows several human activities such as fishing, traveling, and the transporting of large quantities of goods using vessels. Furthermore, its environment host an important and fragile ecosystem that represents a great reservoir and source of food for all living beings. At the same time, the sea has a fundamental role in the climate change, indeed the ocean circulation is a key mechanism of global climate by transporting and storing heat and fresh water around the world. In the last decades the environment in general, and specifically the marine one, has been compromised by several human activities which have undermined both the delicate equilibrium of the ecosystem and the ocean circulation system, activating a dangerous process that involves the extinction risk of some species of sea fish. Moreover, several studies confirm that the extreme climate and meteorological events are strictly related to the modification of the global ocean circulation as well as to the global warming. This latter phenomenon entails the increasing of the global mean sea level that is worrying the population of the small islands. Therefore, it is evident that the sea health is very important for the survival of all humanity. In this context, two concepts were born: the Blue Economy and the Blue Growth, both approaches referring to the use of seas in sustainable way.

Definitively, the sea is a complex environment that includes complex phenomena and assets; as everyone may know, measuring is a fundamental step that allows a deep knowledge of a phenomenon or an asset. The aim of the International Workshop "Metrology for the Sea" is to support the sharing of the recent advances in the field of measurement and instrumentations to be applied for the increasing of knowledge, for protecting and preserving the sea and all assets and phenomena involved. Indeed, every year the Workshop involves hundreds of researchers and people who work in developing instrumentation and measurement methods for the sea activities. Several research topics have been discussed during the edition 2020 of the workshop, such as: the electronic instrumentation, sensors and sensing systems, wireless sensor networks for marine applications, monitoring systems for sea activities, underwater navigation and submarine obstacle detection, pollution detection, measures for marine biology, marine geology, and oceanography.

Five selected papers from MetroSea 2020 are presented in this special issue: one of these is a review paper that illustrates the state of the art and the future perspectives in the field of underwater wireless communications, while the other four papers concern experimental approaches, although applied in different fields.

Leccese and Spagnolo in the paper titled 'State-of-the art and perspectives of underwater optical wireless communications' illustrate the state of art and future perspective of underwater wireless communication using optical waves. This approach is revolutionizing the underwater communications due to the achievable large bandwidth, and low latency levels. Unfortunately, the communication distances are still limited. The authors provide a comprehensive overview of the state of art, 
highlighting which limits distress this technology and which are the possible future developments, especially in military field. It is very interesting their preliminary study to verify the feasibility of a simple, economical, and reliable communication system based on UV-A radiation. Of course, the development of a low-cost underwater communication system with a high transmission rate could have a great impact on the world of the autonomous underwater vehicles.

The scientific contribution presented by Baldo et al., titled 'Remote video monitoring for offshore sea farms: Reliability and availability evaluation and image quality assessment via laboratory tests' describes a preliminary study for the implementation of a remote monitoring system based on video recording for an offshore sea farm. The aim of this work is to ensure a video surveillance infrastructure for supervising breeding cages along with the fish inside them, in order to contrast undesired phenomena like fish poaching as well as cages damages. Since the sea farm are built in open sea, where the weather conditions significantly change from a season to another, the authors focused their inspections on the availability and the reliability of the designed monitoring system, performing several tests in laboratory through a climatic chamber. A specific section is dedicated to the system architecture, where the authors tackle the critical problem of the power supply system. Moreover, it is very interesting that the designed project employs a hardware low-cost and open source such as Raspberry PI.

The other three research papers provide different studies related to the measurements in hydrography. These scientific contributions deal with three different aspects of the hydrographic process. Specifically, the calibration of the echo sounder for acquiring the depth, the enhancement of the positioning system GNSS (Global Navigation Satellite System) and the management of the post processing data.

The scientific contribution by Amoroso and Parente, 'The importance of sound velocity determination for batbymetric survey' presents an inspection on the importance of the sound velocity in water during a hydrographic acquisition. The paper took into consideration four different methodologies for modeling the speed of the sound in sea water. All these approaches need an accurate knowledge of the water density (function of Temperature, Pressure and Salinity) at different depths. The authors reported the impact that inaccurate measurements of these three parameters has on the bathymetric survey results. The experimentation was conducted on real data collected by a hydrographic vessel, while the error propagation was conducted in simulated environment considering several systematic errors on the measurements of the three inspected parameters. This innovative methodology is very promising for the performed inspections.
The paper wrote by Baiocchi et al. titled 'First considerations on post processing kinematic GNSS data during a geophysical oceanographic cruise' concerns the problem of the accurate positioning during a collection of the bathymetric, oceanographic, and geophysical data. The development of oceanographic and geophysical instrumentation, capable to acquire with a high spatial resolution, requires high accuracy positioning systems. In this work the authors carried out several tests on an oceanographic ship applying PPK (Post Process Kinematic) approach to improve the position accuracy provided by the GNSS. The large amount of data acquired by the authors, allowed to validate the performances of the proposed methodology. Indeed, the authors compared the results obtained by PPK processing in the vertical domain with the data registered by several tide gauges located on the surrounding coast. In this work the authors tackled several problems concerning both the acquisition data phase and the different vertical datum used by the tide gauges considered.

Finally, Alcaras et al. presented an interesting work on the post-elaboration of the bathymetric data titled 'From electronic navigational chart data to sea-bottom models: Kriging approaches for the Bay of Pozzuoli. The Electronic Navigational Chart is an electronic map realized by a national hydrographic office and can be used for navigational purposes. The ENC data can be used to build a detailed bathymetric 3D model applying an interpolation method. The authors analyze the performance of different interpolation methods based on the Ordinary and Universal kriging applied to a specific case study: the Bay of Pozzuoli. Hence, the authors employ 11 mathematical semi-variogram models for inspecting the perfomance of these interpolators. However, the cross-validation is used for evaluting the accuracy of each method. The research remarks the good performance of both the Kriging approaches for hydrogrphic purposes and it demonstrates the relevance of the choice of the mathematical model to build the semi-variogram.

I would like to conclude these introductory notes by thanking the authors for their interesting and valuable papers and the reviewers for their indispensable and qualified contributions. Furthermore, I would like to thank the Editor in Chief, prof. Francesco Lamonaca, as his support has been fundamental for accomplishing this Special Issue.

It was a great honour for me to act as Guest Editors for this Special Issue, and I believe that the readers will find this Acta IMEKO issue useful and will be inspired by the themes and methodologies proposed, for continuing the innovation in metrology for the sea.

Silvio Del Pizzo

Section Editor 